\title{
Sodium tanshinone IIA sulfonate improves inflammation, aortic endothelial cell apoptosis, disseminated intravascular coagulation and multiple organ damage in a rat heat stroke model
}

\author{
FANG CHEN, HUIMIN LI, GUOGUO ZHU, XIAOJUAN CHEN and ZHONGZHI TANG
}

Department of Emergency, Wuhan General Hospital of Guangzhou Military Command, Wuhan, Hubei 430070, P.R. China

Received February 4, 2016; Accepted February 20, 2017

DOI: $10.3892 / \mathrm{mmr} .2017 .6573$

\begin{abstract}
The aim of the present study was to investigate the effects of sodium tanshinone IIA sulfonate (STS) on inflammatory responses, aortic endothelial cell apoptosis, disseminated intravascular coagulation (DIC) and multiple organ damage in an animal model of classic heat stroke (CHS). The rats in the heat stroke (HS) and STS-treated heat stroke (STS-HS) groups were placed into a pre-warmed animal temperature controller (ATC) at $35^{\circ} \mathrm{C}$. The moment at which the rectal temperature reached $43.5^{\circ} \mathrm{C}$ was considered as the time of onset of HS. In the HS groups, the rats were removed from the ATC and allowed to recover at $26^{\circ} \mathrm{C}$ for $0,2,6$ or $12 \mathrm{~h}$. In the STS-HS groups, the rats received femoral vein injections of $5-40 \mathrm{mg} / \mathrm{kg}$ STS immediately following the onset of HS and were subsequently placed at a temperature of $26^{\circ} \mathrm{C}$ to recover for $6 \mathrm{~h}$. In the present study, the serum levels of tumor necrosis factor (TNF)- $\alpha$, interleukin (IL)-1 $\beta$ and IL- 6 were assessed using ELISA, and the numbers of apoptotic aortic endothelial cells were investigated using terminal deoxynucleotidyl transferase deoxyuridine triphosphate nick-end labeling combined with immunofluorescence. In the HS groups, the serum levels of TNF- $\alpha$, IL-1 $\beta$ and IL-6, as well as the numbers of apoptotic aortic endothelial cells were increased compared with the normothermic control group. Additionally, the plasma prothrombin time, activated partial thromboplastin time and D-dimer level were significantly increased in the HS group compared with the normothermic control group following recovery for $6 \mathrm{~h}$. By contrast, the platelet count was decreased in the HS group compared with the normothermic control group. The serum levels of creatinine, blood urea nitrogen, alanine aminotransferase, aspartate aminotransferase, alkaline phosphatase and lactate dehydrogenase were increased
\end{abstract}

Correspondence to: Professor Zhongzhi Tang, Department of Emergency, Wuhan General Hospital of Guangzhou Military Command, 627 Wuluo Road, Wuhan, Hubei 430070, P.R. China E-mail: zhzhtang@outlook.com

Key words: heat stroke, sodium tanshinone IIA sulfonate, inflammatory, disseminated intravascular coagulation, multiple organ damage and histopathological damage to multiple organs was observed in the HS group following recovery for $6 \mathrm{~h}$. In the STS-HS groups, cytokine levels and apoptotic aortic endothelial cell numbers were reduced compared with the HS group after $6 \mathrm{~h}$ recovery. STS $(40 \mathrm{mg} / \mathrm{kg}$ ) treatment additionally improved the serum levels of organ injury indicators and plasma indicators of coagulopathy, and prevented histopathological damage to multiple organs. These findings demonstrated that STS treatment may ameliorate multiple organ damage by attenuating inflammatory responses, aortic endothelial cell apoptosis and DIC in CHS. These results suggested that STS may hold potential as an alternative therapeutic strategy for the treatment of patients with HS.

\section{Introduction}

Heat stroke (HS), which results from exposure to a high environmental temperature [classic heat stroke (CHS)] or strenuous exercise [exertional heat stroke (EHS)], is charac terized by a core body temperature (Tc) $>40^{\circ} \mathrm{C}$ and neurological abnormalities. CHS and EHS can lead to multiple organ dysfunction syndrome, which is associated with a systemic inflammatory response and disseminated intravascular coagulation (DIC) (1-6). The mortality rate in patients with HS is increasing, and $\sim 30 \%$ of survivors experience permanent deficits in neurological and peripheral tissue function $(3,7,8)$. A previous study demonstrated that the majority of CHS reactions are mimicked by exposing anesthetized rats to a high ambient temperature (Ta) (9). In these rats, arterial hypotension, hyperpyrexia, a hypercoagulable state, activated inflammation and tissue injury occurred during HS (10-12). Although more research has been undertaken in recent years, specific and effective therapies for HS are required.

Sodium tanshinone IIA sulfonate (STS; Fig. 1A) is a water-soluble derivative of tanshinone IIA. The latter is one of the primary lipophilic components extracted from the dry root or rhizome of Salvia miltiorrhiza Bge, termed 'Danshen' in traditional Chinese medicine (13). Tanshinone IIA has poor water solubility; therefore, STS was developed to increase the bioavailability and has been successfully used to treat patients with cardiovascular disorders. STS has been reported to have a wide range of pharmacological activities, including anti-inflammatory properties (14), antioxidant capacity (15) and the ability to inhibit apoptosis $(16,17)$. 
Furthermore, STS has been demonstrated to suppress cardiomyocyte hypertrophy (18) and protect human vascular endothelial cells in vivo (19). Therefore, HS-induced inflammatory and endothelial cell injuries may be reduced by STS treatment. Proinflammatory cytokines and endothelial cell injury have been demonstrated to initiate coagulation disorders and ultimately lead to DIC in HS $(1,20)$. HS-induced DIC and multiple organ damage may also be reduced by STS treatment.

To evaluate this hypothesis, the present study assessed the therapeutic effects of STS treatment on inflammation, aortic endothelial cell apoptosis, DIC and multiple organ damage in a HS rat model.

\section{Materials and methods}

Animals. A total of 72 adult male Sprague-Dawley rats aged 42-47 days and weighing 200-260 g were obtained from the Animal Resource Center of Hubei (Wuhan, China). The animals were housed individually at a Ta of $25 \pm 1^{\circ} \mathrm{C}$, a relative humidity of $50 \pm 10 \%$ and a 12 -h light/dark cycle for 1 week prior to the start of the experiments. Pelleted rat feed and tap water were provided ad libitum. The experimental protocol was approved by the Animal Resource Center of Hubei. Animal care and experiments were conducted according to the National Institutes of Health Guidelines for the Use of Laboratory Animals (21). The rats were anesthetized by intraperitoneal injections of $50 \mathrm{mg} / \mathrm{kg}$ pentobarbital sodium (Shanghai Haling Biotechnology Co., Ltd., Shanghai, China). The anesthetic administration was completed when the corneal reflex and pain reflexes induced by tail pinch were abolished in the rats. Adequate anesthesia was maintained throughout the course of all experiments.

Induction of HS and experimental design. HS was induced by placing the rats in a warm blanket in an animal temperature controller (ATC; SS-20-2, Huaibei Zhenghua Biological Instrument Equipment Co., Ltd., Huaibei, China) preset to $35^{\circ} \mathrm{C}$. To avoid burns, a towel was placed between the animals and the warm blanket.

The rats were randomly divided into the following groups. In the normothermic control (NC) group, 8 rats were placed into a temperature-controlled room $\left(26^{\circ} \mathrm{C}\right)$ following the administration of anesthesia throughout the entire experiment. In the heat stroke (HS) groups, the Tc (represented by the rectal temperature) of 32 rats were monitored every 5 min following the administration of anesthesia until HS onset. When the Tc reached $43.5^{\circ} \mathrm{C}$, which was considered the time of onset of $\mathrm{HS}$, the rats were removed from the ATC and allowed to recover at $26^{\circ} \mathrm{C}$ for $0,2,6$ or $12 \mathrm{~h}$ (HS-0, HS-2, HS-6 and HS-12 groups, respectively). In the STS-treated heat stroke (STS-HS) groups, 32 rats were exposed to the same heat treatment described above. Immediately following the onset of $\mathrm{HS}$, the rats received intravenous injections of $5,10,20$ or $40 \mathrm{mg} / \mathrm{kg}$ body weight STS (Shanghai No. 1 Biochemical-Pharmaceutical Co., Ltd, Shanghai, China; groups STS-5, STS-10, STS-20 and STS-40, respectively) via the femoral vein and were placed at a temperature of $26^{\circ} \mathrm{C}$ to recover for $6 \mathrm{~h}$. After recovering for the set time, each group of animals was subjected to the following measurements: Serum levels of interleukin-1 $\beta$ (IL-1 $\beta$ ), tumor necrosis factor- $\alpha$ (TNF- $\alpha$ ), and interleukin-6 (IL-6); apoptotic endothelial cells in the aorta; plasma prothrombin time (PT), activated partial thromboplastin time (aPTT), platelet count and D-dimer; serum levels of blood urea nitrogen (BUN), creatinine $(\mathrm{Cr})$, alanine aminotransferase (ALT), aspartate aminotransferase (AST), alkaline phosphatase (ALP) and lactate dehydrogenase (LDH); and histological changes in the major organs.

Cytokine measurements. The serum levels of TNF- $\alpha$, IL-6, and IL-1 $\beta$ were measured using commercially available ELISA kits (catalog no. RTA00 for TNF- $\alpha$; HS600B for IL-6; RLB00 for IL-1 $\beta$; R\&D Systems, Inc., Minneapolis, MN, USA) according to the manufacturer's protocol.

Biochemical analysis. Whole blood was obtained from the abdominal aorta. The serum levels of Cr, BUN, ALT, AST, ALP, and LDH were determined with an automatic biochemical analyzer (DXC-800; Beckman Coulter, Inc., Brea, CA, USA). The plasma PT and aPTT, and D-dimer concentration were measured using an automated coagulation instrument (ACL-TOP; Beckman Coulter, Inc.). The platelet count was determined using an automated blood cell counting instrument (XT-4000i; Sysmex Corporation, Kobe, Japan).

Histological examination. Tissues specimens from livers, kidneys, adrenal glands, small intestines, spleens, and lungs were fixed in $4 \%$ paraformaldehyde, embedded in paraffin, sectioned at $4-\mu \mathrm{m}$ thickness, stained with hematoxylin and eosin, and subsequently imaged under a light microscope with digital camera system (BX51; Olympus Corporation, Tokyo, Japan).

Combined terminal deoxynucleotidyl transferase-mediated dUTP nick-end labeling (TUNEL)/CD31 immunofluorescence assay. For the detection and quantification of apoptotic endothelial cells in aortic sections, TUNEL technology (In Situ Cell Death Detection kit; Roche Applied Science, Madison, WI, USA) was used in combination with immunofluorescence for CD31. The paraffin-embedded sections were stained with a primary rabbit polyclonal anti-CD31 antibody (1:50 dilution; catalog no. ab28364; Abcam, Cambridge, UK) followed by incubation with a Texas Red-conjugated goat anti-rabbit IgG secondary antibody (1:100 dilution; catalog no. BA1032; Wuhan Boster Biological Technology, Ltd., Wuhan, China) to visualize the endothelial cell layer. Briefly, sections were blocked with normal goat serum (Wuhan Boster Biological Technology, Ltd.) for $30 \mathrm{~min}$ at room temperature prior to immunofluorescence staining. The sections were incubated with anti-CD31 antibody overnight at $4^{\circ} \mathrm{C}$. Subsequently, after washing with PBS containing $0.05 \%$ Tween-20, the secondary antibody was applied to the sections for $1 \mathrm{~h}$ at $20-37^{\circ} \mathrm{C}$. The aortic segments embedded in paraffin were then used to detect TUNEL-positive apoptotic cells, according to the manufacturer's protocol. The nuclei were counterstained with 4,6-diamidino-2-phenylindole (DAPI). The percentage of apoptotic endothelial cells was calculated as the proportion of TUNEL/DAPI/CD31-positive cells out of total DAPI/CD31-positive cells. 
A<smiles>Cc1c(S(N)(=O)=O)oc2c1C(=O)C(=O)c1c-2ccc2c1CCCC2(C)C</smiles>

C

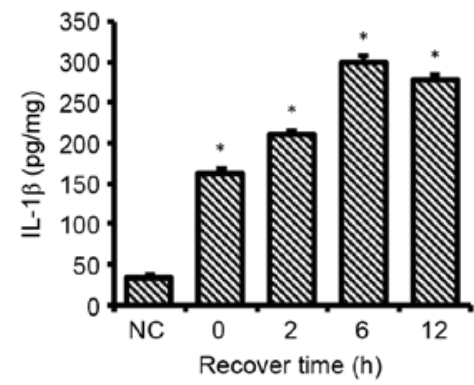

D

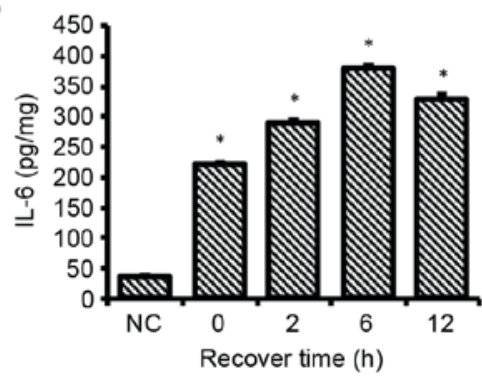

B
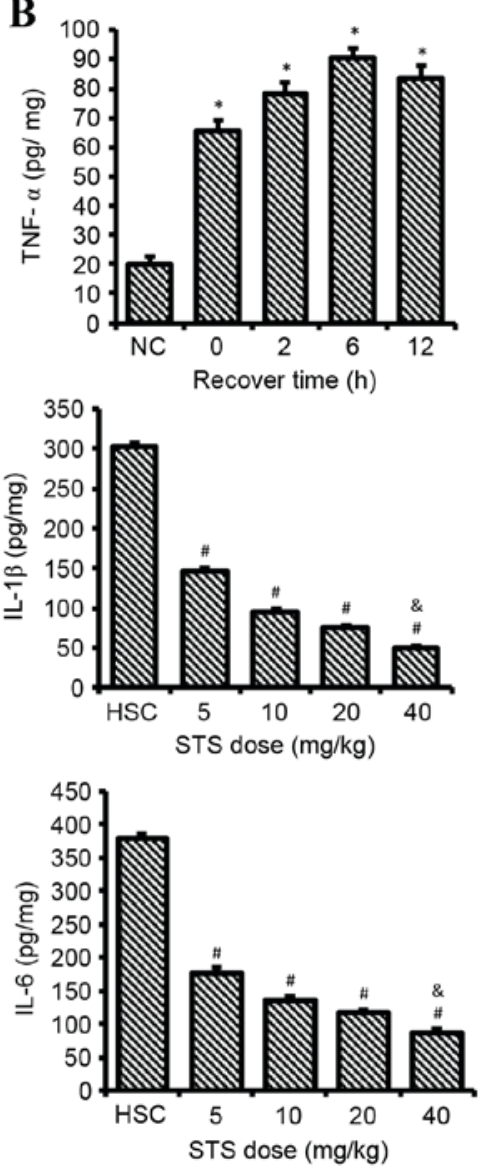

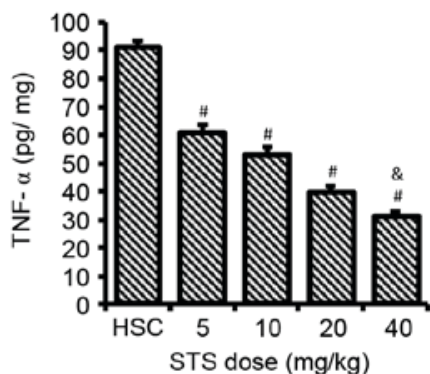

STS dose $(\mathrm{mg} / \mathrm{kg})$

Figure 1. STS decreases the levels of TNF- $\alpha$, IL-1 $\beta$ and IL-6 in serum of HS model rats. (A) Molecular structure of sodium tanshinone IIA sulfonate. The serum levels of (B) TNF- $\alpha$, (C) IL-1 $\beta$ and (D) IL-6 in all groups of rats. Data are presented as the mean \pm standard deviation. ${ }^{*} \mathrm{P}<0.05$ vs. NC; ${ }^{\#} \mathrm{P}<0.05 \mathrm{vs}$. HSC; ${ }^{\&} \mathrm{P}<0.05$ vs. 5,10 and $20 \mathrm{mg} / \mathrm{kg}$ STS. HSC, heat shock control; HS, heat shock; TNF- $\alpha$, tumor necrosis factor- $\alpha$; IL, interleukin; STS, sodium tanshinone IIA sulfonate; NC, normothermic control.

Statistical analysis. Data are expressed as mean \pm standard deviation and were analyzed by one-way analysis of variance followed by least significant difference post hoc test (equal variances) or Dunnett's T3 post hoc test (unequal variances). All analyses were performed using SPSS software version 19.0 (IBM SPSS, Armonk, NY, USA). P<0.05 was considered to indicate a statistically significant difference.

\section{Results}

STS reduces the levels of TNF- $\alpha, I L-1 \beta$ and IL-6 in HS. Clear differences were observed in the serum levels of TNF- $\alpha$ (Fig. 1B), IL-1 $\beta$ (Fig. 1C) and IL-6 (Fig. 1D) between the NC, HS and STS-HS groups. The serum levels of TNF- $\alpha$, IL- $1 \beta$ and IL- 6 in all the HS-0, HS-2, HS- 6 and HS-12 groups were significantly increased compared with the $\mathrm{NC}$ group. The serum levels of TNF- $\alpha$, IL- $1 \beta$ and IL- 6 were greatest in the HS-6 group compared with the other HS groups. Compared to rats that received no STS, treatment with $5-40 \mathrm{mg} / \mathrm{kg}$ STS significantly attenuated the increased serum levels of IL-1 $\beta$, TNF- $\alpha$ and IL- 6 after HS following recovery for $6 \mathrm{~h}$ $(\mathrm{P}<0.05)$. Furthermore, the serum levels of IL-1 $\beta$, TNF- $\alpha$ and IL-6 were maintained at an extremely low level in the rats treated with STS at $40 \mathrm{mg} / \mathrm{kg}$ compared with the $5-20 \mathrm{mg} / \mathrm{kg}$ groups $(\mathrm{P}<0.05)$
STS reduces the number of apoptotic aortic endothelial cells in HS. Significant differences were observed in the number of apoptotic aortic endothelial cells between the NC, HS and STS-HS groups. The number of apoptotic cells in the HS-0, HS-2, HS-6 and HS-12 groups were significantly increased compared with the NC group $(\mathrm{P}<0.05)$. Numbers in the HS-6 group were increased compared with the other HS groups. Treatment with $5-40 \mathrm{mg} / \mathrm{kg}$ STS significantly attenua ted the increased number of apoptotic aortic endothelial cells following HS after recovery for $6 \mathrm{~h}(\mathrm{P}<0.05$; Fig. 2A). Furthermore, the number of apoptotic cells was maintained at an extremely low level in the rats treated with $40 \mathrm{mg} / \mathrm{kg} \mathrm{STS}$, which was significantly lower than in the other STS-treated groups ( $\mathrm{P}<0.05$; Fig. 2B).

STS attenuates DIC in HS. Significant differences were observed in the plasma PT (Fig. 3A), aPTT (Fig. 3B), platelet counts (Fig. 3C) and D-dimer levels (Fig. 3D) in the NC, HS-6 and STS-6-40 group (40 mg/kg STS-treated heat stroke group with recovery for $6 \mathrm{~h}$ ). The plasma PT, aPTT, and D-dimer values in the HS-6 group were significantly increased compared with the NC group. By contrast, the platelet count was significantly reduced in the HS-6 group compared with the NC group. In addition, $40 \mathrm{mg} / \mathrm{kg}$ STS treatment of the HS-6 group attenuated the HS-induced increased plasma 
A
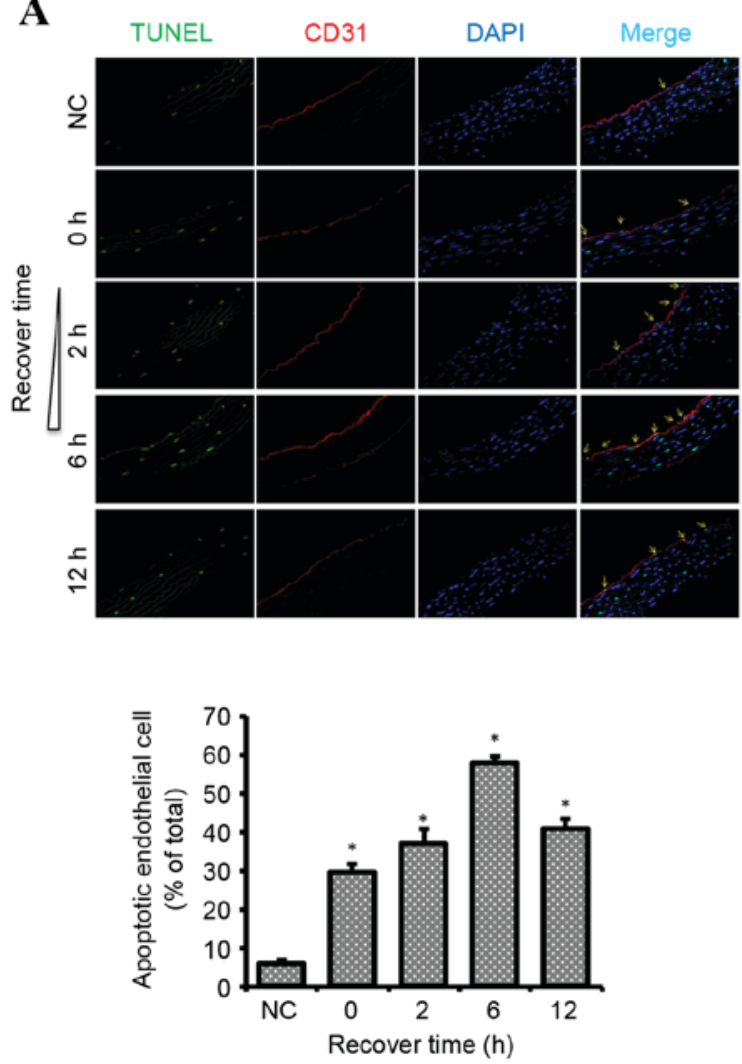

B
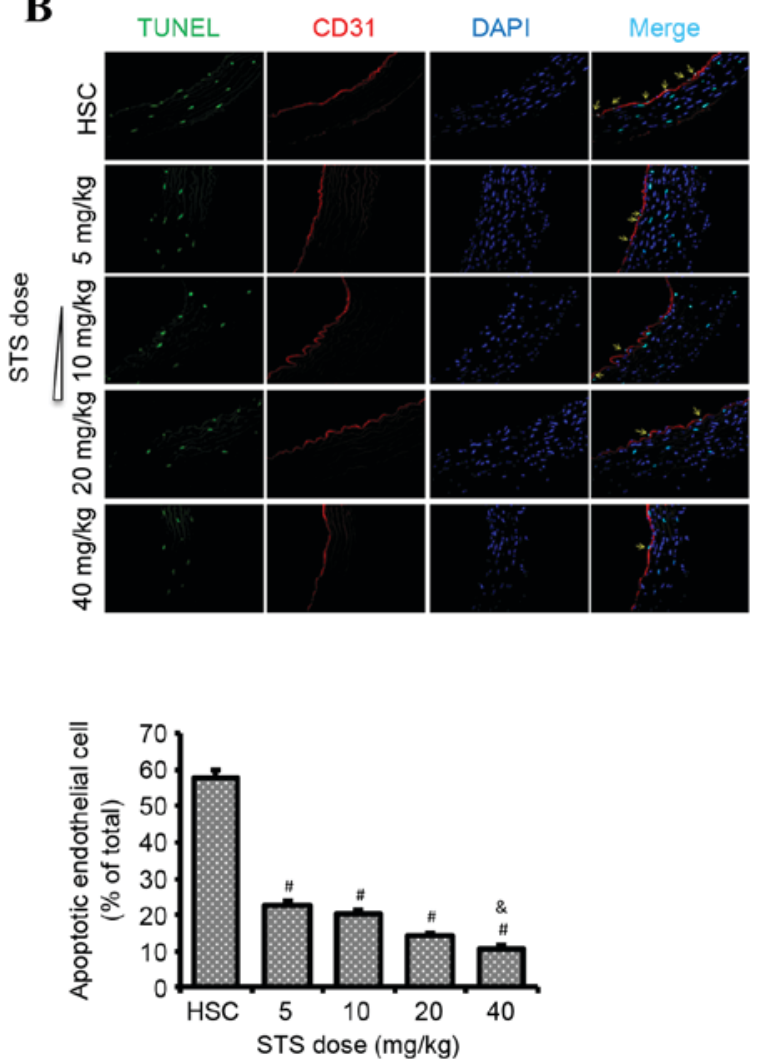

Figure 2. HS-induced apoptosis of aortic endothelial cells is inhibited by STS. Representative micrographs and quantification of apoptosis of aortic endothelial cells at various (A) recovery times and (B) STS doses, as assessed by combined TUNEL (green)/CD31 (red) immunofluorescence assay and counterstaining with DAPI (blue) for nuclei. Apoptotic endothelial cells exhibit combined green/blue/red staining (yellow arrows). Magnification, $\mathrm{x} 400$. Data are presented as the mean \pm standard deviation. ${ }^{*} \mathrm{P}<0.05$ vs. NC; ${ }^{\#} \mathrm{P}<0.05$ vs. HSC; ${ }^{\circledR} \mathrm{P}<0.05$ vs. 5,10 and $20 \mathrm{mg} / \mathrm{kg}$ STS. HSC, heat shock control; HS, heat shock; STS, sodium tanshinone IIA sulfonate; TUNEL, terminal deoxynucleotidyl transferase-mediated dUTP nick-end labeling; NC, normothermic control.

levels of PT, aPTT, and D-dimer and the decreased platelet count $(\mathrm{P}<0.01)$.

The histopathological findings revealed DIC in the vital organs of the HS-6 group. Intravascular thrombus formation was observed in small- and medium-sized blood vessels, and interstitial space hemorrhage was observed in the lungs, kidneys, adrenal glands and livers. However, hemorrhage and thrombosis were significantly alleviated in the STS-6-40 group compared with the HS-6 group (Fig. 3E).

STS attenuates multiple organ damage in HS. Significant differences were observed in serum levels of ALT (Fig. 4A), AST (Fig. 4B), ALP (Fig. 4C), BUN (Fig. 4D), Cr (Fig. 4E), and LDH (Fig. 4F) in the NC, HS-6 and STS-6-40 groups. The serum levels of ALT, AST, ALP, BUN, Cr and LDH in the HS-6 group were significantly increased compared with the NC group. However, $40 \mathrm{mg} / \mathrm{kg}$ STS treatment significantly attenuated the HS-induced increased serum levels of these components $(\mathrm{P}<0.01)$.

The histopathological findings revealed unremarkable damage to the liver, spleen, lung, kidney, and small intestines in the NC group. The damage observed in the major organs of the HS-6 group was extensive; however, a marked decrease in this damage was observed in the STS-6-40 group. The injury to the liver was multifocal in the HS-6 group, including hepatocellular architecture disruption, hepatic cell degenerative changes, hepatic sinusoid congestion and/or hemorrhage, thrombi, and increased inflammatory cells. The architecture of spleen tissues had become disordered, and the boundary between the red and white pulp was unclear in the HS- 6 group. Furthermore, the cellularities of the periarterial lymphatic sheath and the marginal zone were indistinct, although there was extravagated blood in the marginal zone. The lung tissues in the HS-6 group exhibited substantial morphological alterations, including pulmonary edema, alveolar collapse, inflammatory cell infiltration and extensively thickened pulmonary alveolar septa. Furthermore, vascular congestion, hemorrhage, and thrombosis were observed. The kidney injury manifested as tubular epithelial cell edema, hemorrhage, thrombosis, and inflammatory cell infiltration in the HS-6 group. In the small intestines, the bowel walls were swollen and thickened, and the villous architecture exhibited disorder, degeneration, capillary exposure, congestion, and inflammatory cell infiltration. The pathologic impairments of the liver, spleen, lung, kidney and small intestines were significantly alleviated in the STS-6-40 group (Fig. 4G).

\section{Discussion}

The present study demonstrated that HS induced increased inflammatory mediators, aortic endothelial cell apoptosis, DIC and multiple organ damage in an experimental rat model. STS treatment following HS reduced inflammation, aortic endothelial cell apoptosis, DIC and multiple organ damage. 
A

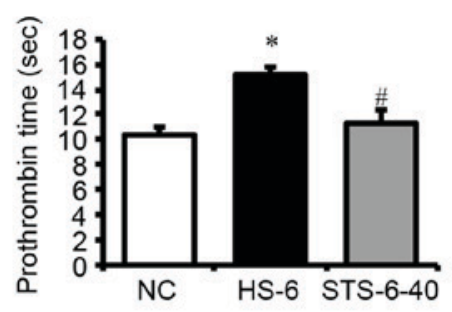

C

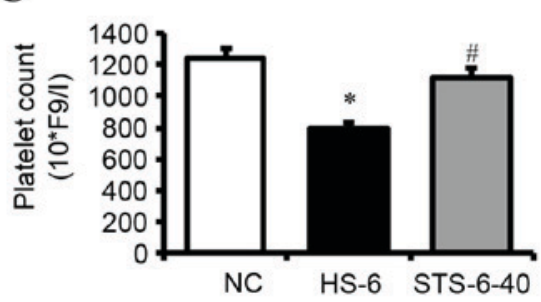

B

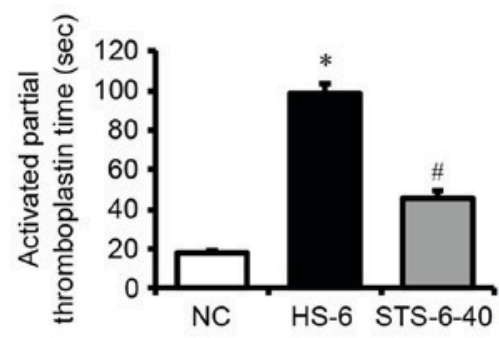

D

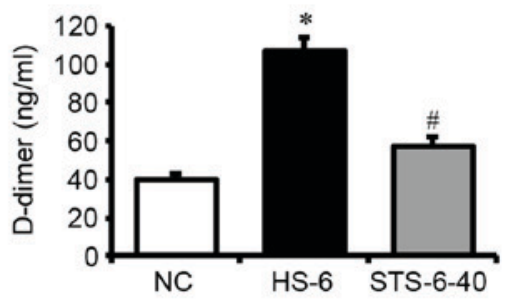

E

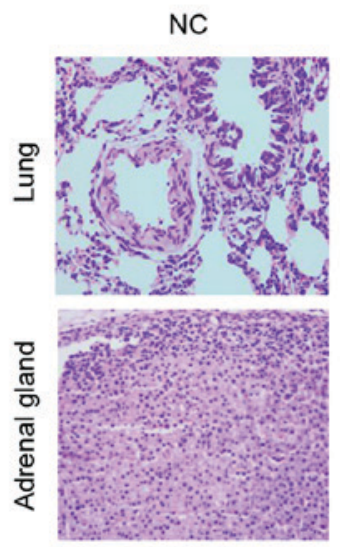

HS-6

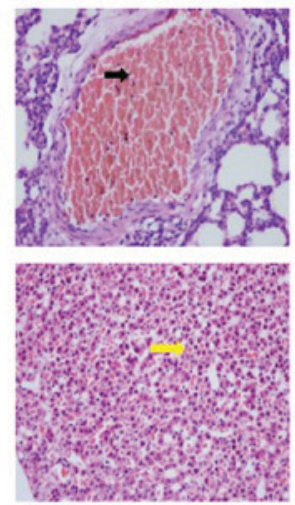

STS-6-40
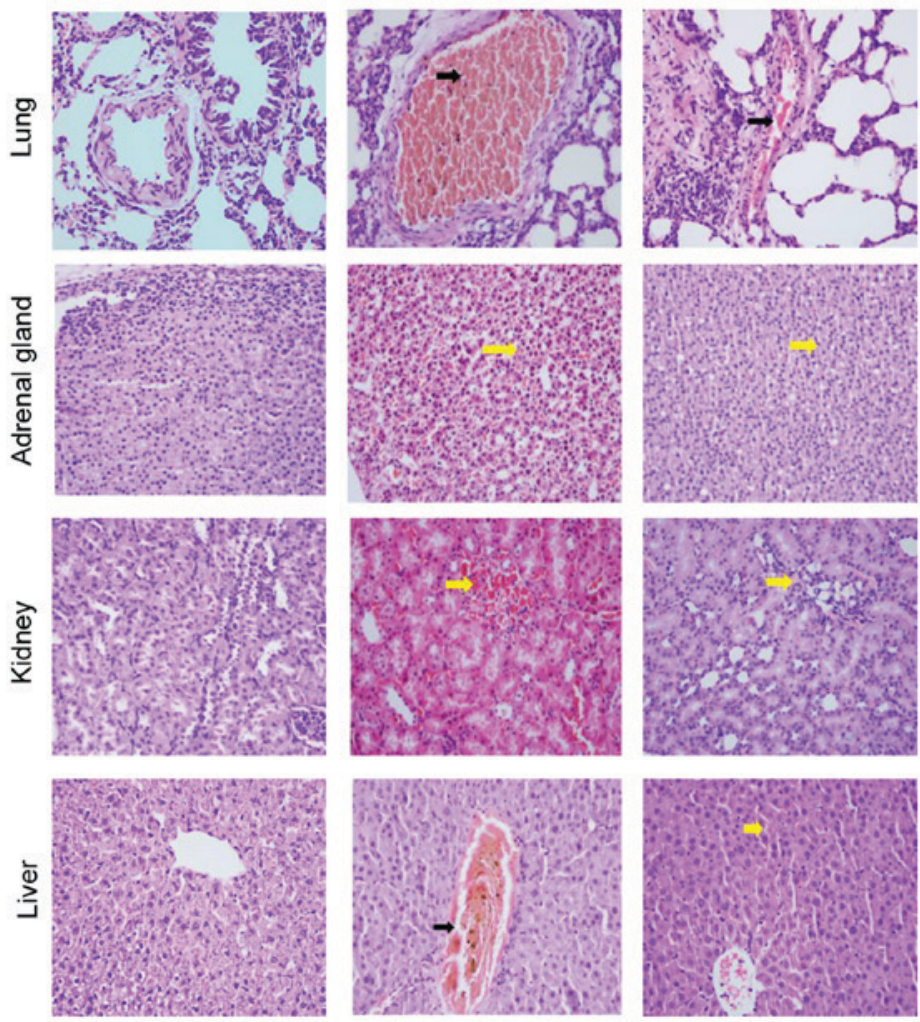

Figure 3. STS negatively regulates HS-induced DIC. Rats were exposed to HS with or without $40 \mathrm{mg} / \mathrm{kg}$ STS, and recovered for $6 \mathrm{~h}$. (A) Prothrombin time, (B) activated partial thromboplastin time, (C) platelet counts and (C) levels of D-dimer in plasma were determined. Data are presented as the mean \pm standard deviation. " $\mathrm{P}<0.05$ vs. NC; "P<0.05 vs. HSC. (E) Representative micrographs of hematoxylin and eosin staining of the lungs, adrenal glands, kidneys and livers. Interstitial space hemorrhage (yellow arrows) in the adrenal gland, kidney and liver. Intravascular thrombus (black arrows) in the central vein of the liver and pulmonary arteriole. Magnification, x400. HS, heat shock; STS, sodium tanshinone IIA sulfonate; NC, normothermic control.

Previous studies have demonstrated that endothelial cell injury is one of the most important features in the pathophysiology of HS $(1,22,23)$. The direct cytotoxic effects of heat and increased inflammatory mediators in HS may induce endothelial cell injury. Activated/injured endothelial cells may result in the release of cytokines, thereby further amplifying the inflammatory response $(3,6,24,25)$. Previous studies demonstrated that the levels of inflammatory factors vary with the recovery time following heat stress. The levels of inflammatory factors are additionally associated with the 
A

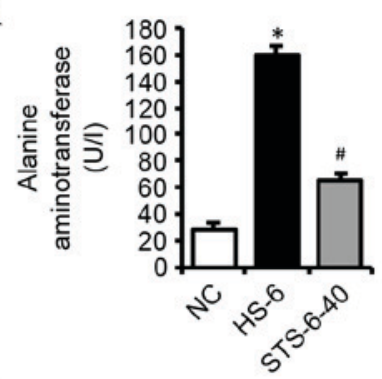

D

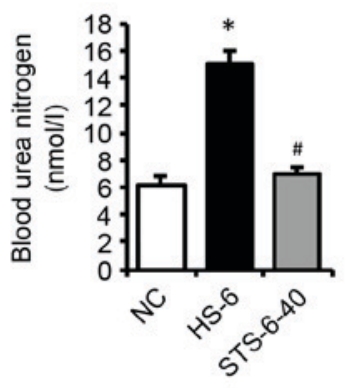

G
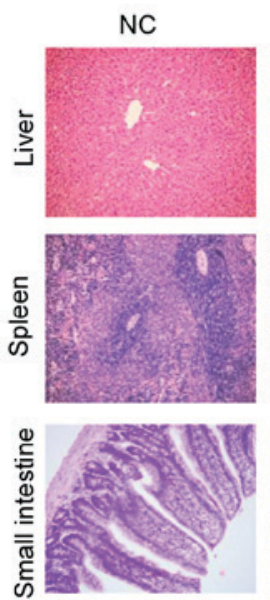

B

E

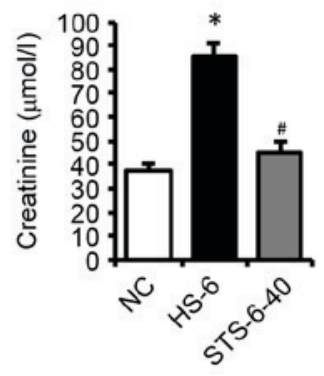

C

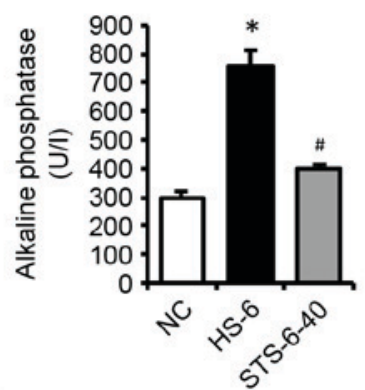

F

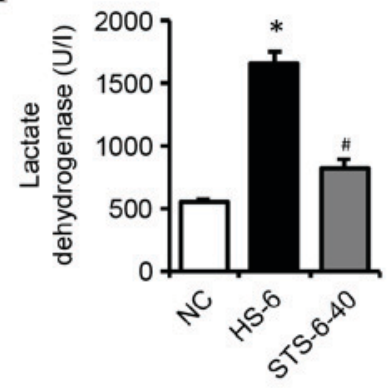

HS-6
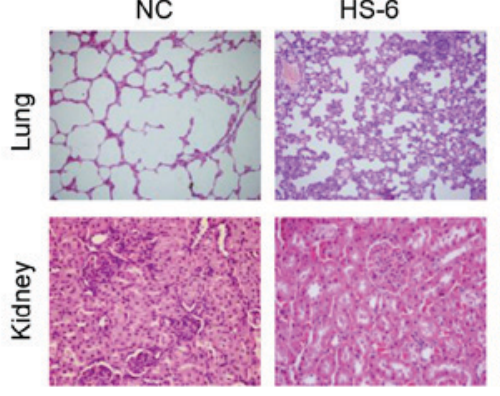

STS-6-40

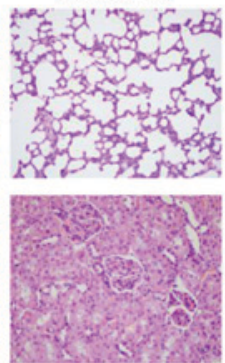

STS-6-40
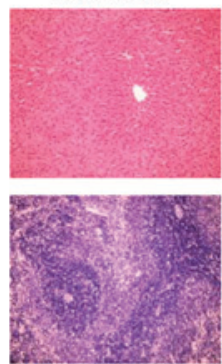

Figure 4. STS prevents heat stroke-induced multiple organ damage. Rats were exposed to HS with or without $40 \mathrm{mg} / \mathrm{kg}$ STS, and recovered for $6 \mathrm{~h}$. Serum levels of (A) alanine aminotransferase, (B) aspartate aminotransferase, (C) alkaline phosphatase, (D) blood urea nitrogen, (E) creatine and (F) lactate dehydrogenase were determined. Data are presented as the mean \pm standard deviation. ${ }^{*} \mathrm{P}<0.05$ vs. NC; ${ }^{~} \mathrm{P}<0.05$ vs. HSC. (G) Representative micrographs of hematoxylin and eosin staining of the livers, spleens, lungs, kidneys, and intestines. Magnification, x200 (liver, spleen, lung, small intestine); magnification, x400 (kidney). NC, normothermic control; HS, heat shock; STS, sodium tanshinone IIA sulfonate.

severity of HS $(1,26,27)$. In these preliminary results, the levels of TNF- $\alpha$, IL- $1 \beta$ and IL- 6 varied with the recovery time following heat exposure, and were highest in the HS-6 group. Previous studies have suggested that intense heat stress may induce endothelial cell apoptosis, and that endothelial cells exhibit significant apoptosis during the acute-phase response to heat stress $(28,29)$. A previous study by $\mathrm{Gu}$ et al (29) demonstrated that in vitro, the rate of apoptosis in human umbilical vein endothelial cells is associated with time after heat treatment, and apoptosis increased most markedly when human umbilical vein endothelial cells were treated at $43^{\circ} \mathrm{C}$ for $2 \mathrm{~h}$, followed by replacement with fresh media and further incubation for $6 \mathrm{~h}$. In the present study, the numbers of apoptotic aortic endothelial cells were highest in the HS-6 group. These results suggested that this time period is likely to represent an important phase in the pathophysiology of HS in which inflammatory mediators, endothelial cell apoptosis and multiple organ damage reach peak levels. Furthermore, in this study, the highest levels of inflammatory mediators and numbers of apoptotic aortic endothelial cells were present at consistent time points; although this may be a coincidence, it demonstrated that inflammatory responses are associated with endothelial cell injury in the pathophysiology of HS (1). Therefore, the HS-6 group was selected as a heat stroke control compared with STS treatment in this study. STS has been reported to have endothelial cell protective abilities and reduce the release of inflammatory factors (30-32). In the present study, STS treatment was demonstrated to dose-dependently decrease the levels of inflammatory mediators and endothelial cell apoptosis.

There is a close association between coagulant activity and the status of endothelial cells (33). Apoptotic endothelial cells exhibit disordered coagulation because of the loss of anticoagulant membrane components, which subsequently leads to procoagulant activation. In addition, apoptotic endothelial cells exhibit decreased expression of coagulation inhibitors, 
such as thrombomodulin, heparin sulfates and tissue factor pathway inhibitor $(25,34)$. Systemic inflammation may additionally contribute to activation of the coagulation system and inhibition of anticoagulant mechanisms and fibrinolysis (35). Imbalance between the procoagulant and anticoagulant systems causes DIC. Previous studies have demonstrated that DIC during HS primarily manifests as prolongation of aPTT and PT, increased D-dimer levels, low platelet counts, and widespread hemorrhage and thrombosis in histological examinations $(22,36-38)$. In the present study, aPTT and PT were prolonged, D-dimer was raised, and platelet counts were decreased in the HS-6 group. In addition, histological examinations revealed intravascular thrombus formation and interstitial space hemorrhage in the vital organs in the HS-6 group. As previously mentioned, STS may reduce the inflammatory response and inhibit endothelial cell apoptosis in HS rats, factors that may lead to DIC. Therefore, STS may attenuate DIC in HS rats. According to these results, STS is considered beneficial in DIC in HS rats.

A complex interplay among the direct cytotoxic effects of heat and the inflammatory and coagulation responses results in multi-organ dysfunction syndrome in HS $(1,3,6)$. Lin et al (39) demonstrated that serum organ injury indicators including Cr, BUN, ALT, AST, ALP and LDH are increased in HS rats. Roberts et al (22) demonstrated that pathological damage to tissues led to vascular congestion, hemorrhage, thrombosis, increased inflammatory cells and disruption of architecture in the vital organs. In the present study, the results of organ damage in the HS-6 group were consistent with previous studies. Multiple organ damage was greatly diminished in the STS-6-40 group. To date, STS has been applied to investigate the effects of HS on DIC and multiple organ damage in rats. STS may attenuate multiple organ damage in CHS by reducing the inflammatory response, aortic endothelial cell apoptosis and DIC. However, there are other potential underlying mechanisms of action in STS treatment of multiple organ damage in CHS, and further studies are required.

In conclusion, the present study demonstrated that STS treatment improves the development of inflammatory processes, aortic endothelial cell apoptosis, DIC and multiple organ damage following CHS. These results suggested that STS treatment may be beneficial for patients with HS.

\section{References}

1. Bouchama A and Knochel JP: Heat stroke. N Engl J Med 346: 1978-1988, 2002.

2. Sucholeiki R: Heatstroke. Semin Neurol 25: 307-314, 2005.

3. Leon LR and Helwig BG: Heat stroke: Role of the systemic inflammatory response. J Appl Physiol (1985) 109: 1980-1988, 2010.

4. al-Mashhadani SA, Gader AG, al Harthi SS, Kangav D, Shaheen FA and Bogus F: The coagulopathy of heat stroke: Alterations in coagulation and fibrinolysis in heat stroke patients during the pilgrimage (Haj) to Makkah. Blood Coagul Fibrinolysis 5: 731-736, 1994.

5. Bouchama A, Bridey F, Hcimmami MM, Lacombe C, al-Shail E, al-Ohali Y, Combe F, al-Sedairy S and de Prost D: Activation of coagulation and fibrinolysis in heatstroke. Thromb Haemost 76: 909-915, 1996.

6. Leon LR: Heat stroke and cytokines. Prog Brain Res 162: 481-524, 2007

7. Levine M, LoVecchio F, Ruha AM, Chu G and Roque P: Influence of drug use on morbidity and mortality in heatstroke. J Med Toxicol 8: 252-257, 2012.
8. Bouchama A: Heatstroke: A new look at an ancient disease. Intensive Care Med 21: 623-625, 1995.

9. Chang CK, Chang CP, Chiu WT and Lin MT: Prevention and repair of circulatory shock and cerebral ischemia/injury by various agents in experimental heatstroke. Curr Med Chem 13: 3145-3154, 2006.

10. Chen YW, Chen SH, Chou W, Lo YM, Hung CH and Lin MT: Exercise pretraining protects against cerebral ischaemia induced by heat stroke in rats. Br J Sports Med 41: 597-602, 2007.

11. Liu CC, Shih MF, Wen YS, Lai YH and Yang TH: Dexamethasone improves heat stroke-induced multiorgan dysfunction and damage in rats. Int J Mol Sci 15: 21299-21313, 2014.

12. Niu KC, Lin MT and Chang CP: Hyperbaric oxygen improves survival in heatstroke rats by reducing multiorgan dysfunction and brain oxidative stress. Eur J Pharmacol 569: 94-102, 2007.

13. Shang Q, Xu H and Huang L: Tanshinone IIA: A promising natural cardioprotective agent. Evid Based Complement Alternat Med 2012: 716459, 2012.

14. Li W, Li J, Ashok M, Wu R, Chen D, Yang L, Yang H, Tracey KJ, Wang P, Sama AE and Wang H: A cardiovascular drug rescues mice from lethal sepsis by selectively attenuating a late-acting proinflammatory mediator, high mobility group box 1 . J Immunol 178: 3856-3864, 2007.

15. Zhou GY, Zhao BL, Hou JW, Ma GE and Xin WJ: Protective effects of sodium tanshinone IIA sulphonate against adriamycin-induced lipid peroxidation in mice hearts in vivo and in vitro. Pharmacol Res 40: 487-491, 1999.

16. Zhang MQ, Zheng YL, Chen H, Tu JF, Shen Y, Guo JP, Yang XH, Yuan SR, Chen LZ, Chai JJ, et al: Sodium tanshinone IIA sulfonate protects rat myocardium against ischemia-reperfusion injury via activation of PI3K/Akt/FOXO3A/Bim pathway. Acta Pharmacol Sin 34: 1386-1396, 2013.

17. Yang R, Liu A, Ma X, Li L, Su D and Liu J: Sodium tanshinone IIA sulfonate protects cardiomyocytes against oxidative stress-mediated apoptosis through inhibiting JNK activation. J Cardiovasc Pharmacol 51: 396-401, 2008.

18. Yang L, Zou X, Liang Q, Chen H, Feng J, Yan L, Wang Z, Zhou D, Li S, Yao S and Zheng Z: Sodium tanshinone IIA sulfonate depresses angiotensin II-induced cardiomyocyte hypertrophy through MEK/ERK pathway. Exp Mol Med 39: 65-73, 2007.

19. Wu TW, Zeng H, Fung KP, Wu J, Pang H, Grey AA, Weisel RD and Wang JY: Effect of sodium tanshinone IIA sulfonate in the rabbit myocardium and on human cardiomyocytes and vascular endothelial cells. Biochem Pharmacol 46: 2327-2332, 1993.

20. Bombeli T, Karsan A, Tait JF and Harlan JM: Apoptotic vascular endothelial cells become procoagulant. Blood 89: 2429-2442, 1997.

21. Clark JD, Baldwin RL, Bayne KA, Manyland R, Brown MJ, Gebhart GF, Gonder JC, Gwathmey JK, Keeling ME, Kohn DF, et al: Guide for the Care and Use of Laboratory Animals. The national academies press. 1996.

22. Roberts GT, Ghebeh H, Chishti MA, Al-Mohanna F, El-Sayed R, Al-Mohanna $F$ and Bouchama A: Microvascular injury, thrombosis, inflammation, and apoptosis in the pathogenesis of heatstroke: A study in baboon model. Arterioscler Thromb Vasc Biol 28: 1130-1136, 2008.

23. Bouchama A, Hammami MM, Haq A, Jackson J and al-Sedairy S: Evidence for endothelial cell activation/injury in heatstroke. Crit Care Med 24: 1173-1178, 1996.

24. Tong H, Wan P, Zhang X, Duan P, Tang Y, Chen Y, Tang L and Su L: Vascular endothelial cell injury partly induced by mesenteric lymph in heat stroke. Inflammation 37: 27-34, 2014.

25. Lovren F and Verma S: Evolving role of microparticles in the pathophysiology of endothelial dysfunction. Clin Chem 59: 1166-1174, 2013.

26. Yamakawa K, Matsumoto N, Imamura Y, Muroya T, Yamada T, Nakagawa J, Shimazaki J, Ogura H, Kuwagata Y and Shimazu T: Electrical vagus nerve stimulation attenuates systemic inflammation and improves survival in a rat heatstroke model. PLoS One 8: e56728, 2013.

27. Leon LR, Blaha MD and DuBose DA: Time course of cytokine, corticosterone, and tissue injury responses in mice during heat strain recovery. J Appl Physiol (1985) 100: 1400-1409, 2006.

28. Brinton MR, Tagge CA, Stewart RJ, Cheung AK, Shiu YT and Christensen DA: Thermal sensitivity of endothelial cells on synthetic vascular graft material. Int J Hyperthermia 28: 163-174, 2012.

29. Gu ZT, Wang H, Li L, Liu YS, Deng XB, Huo SF, Yuan FF, Liu ZF, Tong HS and Su L: Heat stress induces apoptosis through transcription-independent p53-mediated mitochondrial pathways in human umbilical vein endothelial cell. Sci Rep 4: 4469, 2014. 
30. Zhou ZW, Xie XL, Zhou SF and Li CG: Mechanism of reversal of high glucose-induced endothelial nitric oxide synthase uncoupling by tanshinone IIA in human endothelial cell line EA.hy926. Eur J Pharmacol 697: 97-105, 2012.

31. Jang SI, Jeong SI, Kim KJ, Kim HJ, Yu HH, Park R, Kim HM and You YO: Tanshinone IIA from salvia miltiorrhiza inhibits inducible nitric oxide synthase expression and production of TNF-alpha, IL-1beta and IL-6 in activated RAW 264.7 cells. Planta Med 69: 1057-1059, 2003.

32. Jang SI, Kim HJ, Kim YJ, Jeong SI and You YO: Tanshinone IIA inhibits LPS-induced NF-kappaB activation in RAW 264.7 cells: Possible involvement of the NIK-IKK, ERK1/2, p38 and JNK pathways. Eur J Pharmacol 542: 1-7, 2006.

33. Vallet B and Wiel E: Endothelial cell dysfunction and coagulation. Crit Care Med 29 (Suppl 7): S36-S41, 2001.

34. Bouvy C, Gheldof D, Chatelain C, Mullier F and Dogné JM: Contributing role of extracellular vesicles on vascular endothelium haemostatic balance in cancer. J Extracell Vesicles 3: Jul 11, 2014 doi: 10.3402/jev.v3.24400.
35. Schouten M, Wiersinga WJ, Levi $M$ and van der Poll T: Inflammation, endothelium, and coagulation in sepsis. J Leukoc Biol 83: 536-545, 2008.

36. Chen CM, Hou CC, Cheng KC, Tian RL, Chang CP and Lin MT: Activated protein $\mathrm{C}$ therapy in a rat heat stroke model. Crit Care Med 34: 1960-1966, 2006.

37. Ockelford PA and Carter CJ: Disseminated intravascular coagulation: The application and utility of diagnostic tests. Semin Thromb Hemost 8: 198-216, 1982.

38. Sohal RS, Sun SC, Colcolough HL and Burch GE: Heat stroke An electron microscopic study of endothelial cell damage and disseminated intravascular coagulation. Arch Intern Med 122: 43-47, 1968.

39. Lin XJ, Mei GP, Liu J, Li YL, Zuo D, Liu SJ, Zhao TB and Lin MT: Therapeutic effects of melatonin on heatstroke-induced multiple organ dysfunction syndrome in rats. J Pineal Res 50: 436-444, 2011. 